\title{
Ordered linear space of hyperfunctions
}

\author{
Deepthi $\mathrm{A} \mathrm{N}^{1 *}$ and Mangalambal $\mathrm{N} \mathrm{R}^{2}$
}

\begin{abstract}
Mikio Sato introduced the concept of hyperfunctions to explain generalized functions in the mathematical modeling of physical problems. In this paper an order relation is introduced to a subclass of the space of hyperfunctions and also defined cone in it.Some properties of this cone are studied by introducing a topology to it.
\end{abstract}

\section{Keywords}

Hyperfunctions, Cones.

\section{AMS Subject Classification} 46F15,47L07.

1,2 Center for Research in Mathematical Sciences, Department of Mathematics, St.Joseph's College, Irinjalakuda-680121, India.

*Corresponding author: ${ }^{1}$ deepthiakshaya@gmail.com; ${ }^{2}$ thottuvai@gmail.com

Article History: Received 24 January 2019; Accepted 24 May 2019

\section{Contents}

1 Introduction 485

2

3 Main Results 485

4

Conclusion 487

References 488 488

\section{Introduction}

Mikio Sato introduce the idea of hyperfunctions to mention his generalization of the concept of functions. By a hyperfunction he mean an equivalence class of functions satisfying certain conditions.

In this paper we are mainly considering hyperfunctions having bounded exponential growth and having defining function a complex measurable holomorphic function. An order relation is introduced among these type of hyperfunctions using the defining functions of hyperfunctions. Also cone is defined in it.Some properties of this cone are studied. Also an inductive limit topology is defined on it and compare it with the order topology using [2],[3],[4],[5],[6],[7].

\section{Preliminaries}

In this section, we recall some definitions and basic results of hyperfunctions which will be used throughout the paper. We denote the upper and lower half-plane of the complex plane $\mathscr{C}$ by

$$
\begin{aligned}
& \mathscr{C}_{+}=\{z \in \mathscr{C}: I z>0\}, \\
& \mathscr{C}_{-}=\{z \in \mathscr{C}: I z<0\}
\end{aligned}
$$

respectively.

Definition 2.1. [1] For an open interval I of the real line, the open subset $N(I) \subset \mathscr{C}$ is called a complex neighborhood of I, if $I$ is a closed subset of $N(I)$.

We let $N_{+}(I)=N(I) \cap \mathscr{C}_{+}$and $N_{-}(I)=N(I) \cap \mathscr{C}_{-}$.

$\mathfrak{O}(N(I) \backslash I)$ denotes the ring of holomophic functions in $N(I) \backslash$ I.

For a given interval I a function $F(z) \in \mathfrak{O}(N(I) \backslash I)$ can be written as

$$
F(z)= \begin{cases}F_{+}(z) & \text { for } z \in N_{+}(I), \\ F_{-}(z) & \text { for } z \in N_{-}(I)\end{cases}
$$

where $F_{+}(z) \in \mathfrak{O}\left(N_{+}(I)\right)$ and $F_{-}(z) \in \mathfrak{O}\left(N_{-}(I)\right)$, are called upper and lower componet of $F(z)$ respectively. In general the upper and lower component of $F(z)$ need not be related to each other. If they are analytic continuations from each other we call $F(z)$ a global analytic function on $N(I)$ and write $F_{+}(z)=F_{-}(z)=F(z)$

Definition 2.2. [1] Two functions $F(z)$ and $G(z)$ in $\mathfrak{O}(N(I) \backslash$ I) are equivalent if for $z \in N_{1}(I) \cap N_{2}(I)$,

$$
G(z)=F(z)+\phi(z)
$$

, with $\phi(z) \in \mathfrak{O}(N(I))$ where $N_{1}(I)$ and $N_{2}(I)$ are complex neighborhoods of I of $F(z)$ and $G(z)$ respectively. 
Definition 2.3. [1] An equivalence class of functions $F(z) \in$ $\mathfrak{O}(N(I) \backslash I)$ defines a hyperfunction $f(x)$ on I. Which is denoted by $f(x)=[F(z)]=\left[F_{+}(z), F_{-}(z)\right] . F(z)$ is called defining or generating function of the hyperfunction.

The set of all hyperfunctions defined on the interval I is denoted by $\mathfrak{B}(I)$.

$$
\mathfrak{B}(I)=\mathfrak{O}(N(I) \backslash I) \backslash \mathfrak{O}(N(I))
$$

A real analytic function $\phi(x)$ on $I$ is defined by the fact that $\phi(x)$ can analytically be continued to a full neighborhood $U$ containing $I$ i.e. we then have $\phi(z) \in \mathfrak{O}(U)$. For any complex neighborhood $N(I)$ containing $U$ we may then write

$$
\mathfrak{B}(I)=\mathfrak{O}(N(I) \backslash I) \backslash \mathscr{A}(I),
$$

where $\mathscr{A}(I)$ is the ring of all real analytic functions on I.Thus a hyperfunction $f(x) \in \mathfrak{B}(I)$ is determined by a defining function $F(z)$ which is holomorphic in an adjacent(small) neighborhood above and below the interval I, but is only determined upto a real analytic function on $I$.

Definition 2.4. For $F(z)$ it may happen that at $x \in I_{0} \subseteq I$ the limit

$$
\lim _{\varepsilon \rightarrow 0^{+}}\left\{F_{+}(x+i \varepsilon)-F_{-}(x-i \varepsilon)\right\}
$$

exists. A point $x \in I \backslash I_{0}$ is called a singular point and a point $x \in I_{0}$ is called a regular point of the hyperfunction. At a regular point, a hyperfunction $f(x)$ has a function-value as an ordinary function. At a singular point it does not make sense to speak of a function-value of the hyperfunction. Hence

$f(x)=F(x+i 0)-F(x-i 0)=\lim _{\varepsilon \rightarrow 0^{+}}\left\{F_{+}(x+i \varepsilon)-F_{-}(x-i \varepsilon)\right\}$

provided the limit exists.

Example 2.5. [1] Dirac delta function at $x=0$ is represented in terms of hyperfunction as $\delta(x)=\left[\frac{-1}{2 \pi i z}\right]$. Here the defining function is $F(z)=\frac{-1}{2 \pi i z} . \quad F(z)$ is defined except at $z=0$. At $z=0, F(z)$ has an isolated singularity, which is a pole of order 1 . For every real number $x \neq 0$ the limit $\lim _{\varepsilon \rightarrow 0^{+}}\left\{F_{+}(x+i \varepsilon)-F_{-}(x-i \varepsilon)\right\}$ exists and equal to 0 .

Definition 2.6. [1] A real analytic function $\phi(x)$ is a function which is holomorphic in a full neighborhood of the entire real axis i.e. $\phi(x) \in \mathscr{A}(\mathbb{R})$.

Example $e^{x}$. Which is real analytic, it can be analytically continued to the entire function $e^{z}$ holomorphic in the entire complex plane.

Definition 2.7. [1] The hyperfunction $f(x)=[\phi(z)]$, where $\phi(x) \in \mathscr{A}(\mathbb{R})$ is any real analytic function represents the zero hyperfunction. We denote the zero hyperfunction by $O$ since it can be identified with the ordinary zero function.

Two hyperfunctions $f(x), g(x) \in \mathfrak{B}(I)$ can be added. If $f(x)=[F(z)], g(x)=[G(z)]$, define

$$
f(x)+g(x)=[F(z)+G(z)]
$$

- Similarly scalar multiplication can be defined as for any $c \in \mathscr{C}$

$$
c f(x)=c[F(z)]=[c F(z)]
$$

It can be shown that this addition and scalar multiplication is well define on $\mathfrak{B}(I)$

Proposition 2.8. $\mathfrak{B}(I)$ is a linear space over $\mathscr{C}$.

Definition 2.9. [1] If $f(x)=[F(z)] \in \mathfrak{B}(I)$ is a hyperfunction and $\phi(x) \in \mathscr{A}(I)$ is a real analytic function on I,then $\phi(x) f(x) \in \mathfrak{B}(I) . \phi(x) f(x)=[\phi(z) F(z)]$

Definition 2.10. [1] For any given hyperfunction $f(x)=$ $\left[F_{+}(z), F_{-}(z)\right]$ its derivative in the sense of hyperfunction is defined as

$$
\begin{aligned}
& D f(x)=f(x)=\left[\frac{d F_{+}}{d z}, \frac{d F_{-}}{d z}\right] \\
& D^{n} f(x)=f^{(n)}(x)=\left[\frac{d^{n} F_{+}}{d z^{n}}, \frac{d^{n} F_{-}}{d z^{n}}\right]
\end{aligned}
$$

Proposition 2.11. Hyperfunctions are always infinitely differentiable.

Definition 2.12. [1] A hyperfunction $f(x)$ is called holomorphic at $x=a$, if the lower and upper component of the defining function can analytically be continued to a full(two-dimensional) neighborhood of the real point a i.e. the upper/ lower component can analytically be continued across a into the lower/upper half-plane.

Definition 2.13. [1] Consider hyperfunctions depending on a continuous parameter $\alpha$ or an integral parameter $k$. The continuous parameter $\alpha$ varies in some open region $\Omega$ of the complex plane and $\alpha_{0}$ is a limit point of $\Omega$. Integral parameter $k$ may vary in $\mathbb{N}$ or $\mathbb{Z}$. Then $f(x, \alpha)=[F(z, \alpha)], \alpha \in$ $\Omega ; f_{k}(x)=\left[F_{k}(z)\right], k \in \mathbb{N}$ or $\mathbb{Z}$. We say that a family of holomorphic functions $F(z, \alpha)$, or a sequence of holomorphic functions $F_{k}(z)$ defined on a common domain $N \subset \mathscr{C}$ converges uniformly in the interior of $N$ to $F(z)$ as $\alpha \rightarrow \alpha_{0}$, or $k \rightarrow \infty$, respectively if $F(z, \alpha)$ or $F_{k}(z)$ converges uniformly to $F(z)$ in every compact sub domain of $N$. This uniform convergence in the interior of $N$ is also called compact convergence in $N$.

Definition 2.14. [1] Let $f(x)=\left[F_{+}(z), F_{-}(z)\right]$ be defined on I such that, for every $k$, equivalent defining functions $G_{k}(z)$ of $F_{k}(z)$ exist, such that $G_{k_{+}}(z)$ and $G_{k_{-}}(z)$ are uniformly convergent in the interior of $N_{+}(I)$ and $N_{-}(I)$ to $F_{+}(z)$ and $F_{-}(z)$ respectively. Then we write

$$
f(x)=\lim _{k \rightarrow \infty} f_{k}(x)
$$

and say that the sequence of hyperfunctions $f_{k}(x)$ converges in the sense of hyperfunctions to $f(x)$.

Proposition 2.15. If a limit in the sense of hyperfunctions exists, it is unique. 
Definition 2.16. [1] Let $\Sigma_{0}$ be the largest open subset of the real line where the hyperfunction $f(x)=[F(z)]$ is vanishing. Its complement $K_{0}=\mathbb{R} \backslash \Sigma_{0}$ is said to be the support of the hyperfunction $f(x)$ denoted by supp $f(x)$.

Definition 2.17. [1] Let $\Sigma_{1}$ be the largest open subset of the real line where the hyperfunction $f(x)=[F(z)]$ is holomorphic. Its complement $K_{1}=\mathbb{R} \backslash \Sigma_{1}$ is said to be the singular support of the hyperfunction $f(x)$ denoted by singsupp $f(x)$.

For the following let us consider open sets $J=(a, 0) \cup(0, b)$ with some a less than 0 and some $b>0$ and compact subsets $K=\left[a^{\prime}, a^{\prime \prime}\right] \cup\left[b^{\prime}, b^{\prime \prime}\right]$ with $a<a^{\prime} \leq a^{\prime \prime}<0$ and $0<b^{\prime} \leq b^{\prime \prime}<b$. Also consider the following open neighborhoods $[-\delta, \infty)+i J$ and $(-\infty, \delta]+i J$ of $\mathbb{R}_{+}$and $\mathbb{R}_{-}$respec tively for some $\delta>0$ Introduce the subclass $\mathfrak{O}\left(\mathbb{R}_{+}\right)$of hyperfunctions $f(x)=[F(z)]$ on $\mathbb{R}$ satisfying

(i)The support supp $f(x)$ is contained in $[0, \infty)$

(ii) Either the support supp $f(t)$ is bounded on the right by a finite number $\beta>0$ or we demand that among all equivalent defining functions, there is one, $F(z)$ defined in $[-\delta, \infty)+i J$ such that for any compact $K \subset J$ there exist some real constant $M^{\prime}>0$ and $\sigma^{\prime}$ such that $|F(z)| \leq M^{\prime} e^{\sigma^{\prime} \mathfrak{R} z}$ holds uniformly for all $z \in[0, \infty)+i K$

Because supp $f(x) \subset \mathbb{R}_{+}$and since the singular support singsuppf is a subset of the support, we have singsupp $f \subset$ $\mathbb{R}_{+}$. Therefore $f(x)$ is a holomorphic hyperfunction for all $x<0$. Moreover, the fact that $F_{+}(x+i 0)-F_{-}(x-i 0)=0$ for all $x<0$ shows that $F(z)$ is real analytic on the negative part of the real axis. Hence $f(x) \in \mathfrak{O}\left(\mathbb{R}_{+}\right)$implies that

$$
\chi_{(-\varepsilon, \infty)} f(t)=f(t)
$$

for any $\varepsilon>0$.

Definition 2.18. [1] We call the subclass of hyperfunctions $\mathfrak{O}\left(\mathbb{R}_{+}\right)$the class of right sided originals.

In the case of an unbounded support suppf $(t)$, let $\sigma=$ inf $\sigma^{\prime}$ be the greatest lower bound of all $\sigma^{\prime}$ where the infimum is taken over all $\sigma^{\prime}$ and all equivalent defining functions satisfying (ii). This number $\sigma_{-}=\sigma_{-}(f)$ is called the growth index of $f(t) \in \mathbb{R}_{+}$. It has the properties

(i) $\sigma_{-} \leq \sigma^{\prime}$

(ii) For every $\varepsilon>0$ there is a $\sigma^{\prime}$ with $\sigma_{-} \leq \sigma^{\prime} \leq \sigma_{-}+\varepsilon$ and an equivalent defining function $F(z)$ such that $|F(z)| \leq$ $M^{\prime} e^{\sigma^{\prime} \mathfrak{R} z}$ uniformly for all $z \in[0, \infty)+i K$.

In the case of a bounded support supp $f(t)$,we set $\sigma_{-}(f)=$ $-\infty$
Similarly, we introduce the class $\mathfrak{O}\left(\mathbb{R}_{-}\right)$of hyperfunctions specified by

(i) The support supp $(t)$ is contained in $\mathbb{R}_{-}=(-\infty, 0]$

(ii) Either the support suppf(t) is bounded on the left by a finite number $\alpha<0$, or we demand that among all equivalent defining functions there is one, denoted by $F(z)$ and defined in $(-\infty, \delta]+i J$ such that for any compact subset $K \subset J$ there are some real constants $M^{\prime \prime}>0$ and $\sigma^{\prime \prime}$ such that $|F(z)| \leq M^{\prime \prime} e^{\sigma^{\prime \prime} \Re z}$ holds uniformly for $z \in(-\infty, 0]+i K$.

Definition 2.19. [1] The set $\mathfrak{O}\left(\mathbb{R}_{-}\right)$is said to be the class of left-sided originals.

In the case of an unbounded support let $\sigma_{+}=\sup \sigma^{\prime \prime}$ be the least upper bound of all $\sigma^{\prime \prime}$, where the supremum is taken over all $\sigma^{\prime \prime}$ and all equivalent defining functions satisfying (ii). The number $\sigma_{+}=\sigma_{+}(f)$ is called the growth index of $f(t) \in \mathfrak{O}\left(\mathbb{R}_{-}\right)$. It has the properties

(i) $\sigma^{\prime \prime} \leq \sigma_{+}$

(ii) For every $\varepsilon>0$ there is a $\sigma^{\prime \prime}$ such that $\sigma_{+}-\varepsilon \leq \sigma^{\prime \prime} \leq \sigma_{+}$ and a definig function $F(z)$ such that $|F(z)| \leq M^{\prime \prime} e^{\sigma^{\prime \prime} \mathfrak{R} z}$ uniformly for $z \in(-\infty, 0]+i K$.

If the support supp $f(t)$ is bounded, we set $\sigma_{+}(f)=+\infty$

With a left-sided original $g(t) \in \mathfrak{O}\left(\mathbb{R}_{-}\right)$with growth index $\sigma_{+}(g)$ and a right-sided original $f(t) \in \mathfrak{O}\left(\mathbb{R}_{+}\right)$with growth index $\sigma_{-}(f)$ form the hyperfunction $h(t)=g(t)+h(t)$ whose support is now the entire real axis.

Definition 2.20. [1] Hyperfunctions of the subclass $\mathfrak{O}\left(\mathbb{R}_{+}\right)$ are said to be of bounded exponential growth as $x \rightarrow \infty$ and hyperfunctions of the subclass $\mathfrak{O}\left(\mathbb{R}_{-}\right)$are said to be of bounded exponential growth as $x \rightarrow-\infty$.

Definition 2.21. [1] An ordinary function $f(t)$ is called of bounded exponential growth as $t \rightarrow \infty$, if there are some real constants $M^{\prime}>0$ and $\sigma^{\prime}$ such that $|f(t)| \leq M^{\prime} e^{\sigma^{\prime} t}$ for sufficently large $t$. It is called of bounded exponential growth as $t \rightarrow-\infty$, if there are some real constants $M^{\prime \prime}>0$ and $\sigma^{\prime \prime}$ such that $|f(t)| \leq M^{\prime \prime} e^{\sigma^{\prime \prime} t}$, for sufficently negative large $t$ A function or a hyperfunction is of bounded exponential growth, if it is of bounded exponential growth for $t \rightarrow-\infty$ as well as for $t \rightarrow \infty$.

\section{Main Results}

In this section defined a cone in the linear space of hyperfunctions and some of its properties are investigated.

Note: Let the space of real valued hyperfunctions be denoted by $\mathfrak{B}_{\mathbb{R}}(I)$.Then $\mathfrak{B}_{\mathbb{R}}(I)$ is a linear space over $\mathbb{R}$

Definition 3.1. Let $f(x), g(x) \in \mathfrak{B}_{\mathbb{R}}(I)$ where $f(x)=[F(z)]$, $g(x)=[G(z)]$. Define the order relation' $\leq$ by $f \leq g$ if 
$f(x) \leq g(x)$ i.e. $F(z) \leq G(z)$ and it holds for every other representatives from the corresponding equivalence classes .Then ${ }^{\prime} \leq$ is a partial order on $\mathfrak{B}_{\mathbb{R}}(I)$.

Proposition 3.2. $\mathfrak{B}_{\mathbb{R}}(I)$ is an ordered linear space

Note: Here onwards consider I as a subset of $\mathbb{R}_{+}=(0, \infty)$. Let $\mathfrak{B}_{B}^{M}(I)$ denotes the linear space of hyperfunctions of bounded exponential growth and has defining function a complex measurable holomorphic function.

Let $\bar{N}(0, n)=\{z \in \mathscr{C}:|z| \leq n\}$,i.e. $\bar{N}(0, n)$ denotes the closed complex neighborhood of 0 .

Definition 3.3. Let $N$ be a complex neighborhood of I. For $n=1,2, \ldots$ define

$$
K_{n}=\bar{N}(0, n) \cap\left\{z:|z-w| \geq \frac{1}{n}, \forall w \in \mathscr{C} \backslash N\right\}
$$

Then $\left\{K_{n}\right\}$ has thefollowing properties

i) $K_{n}$ is compact

ii) $K_{n} \subseteq K_{n+1}$

iii)If $K \subseteq N$ is compact then $K \subseteq K_{n}$ for sufficently large $n$.

On each $K_{n}$ and $f(x)=[F(Z)] \in \mathfrak{B}(I)$ define

$$
\begin{array}{r}
\beta_{K_{n}, m}(f(x))=\sup \left\{\left|\frac{d^{m}}{d z^{m}} F(z)\right|: \forall F(z) \in[F(z)], z \in K_{n}\right\}, \\
m=0,1,2, \ldots
\end{array}
$$

Then $\left\{\beta_{K_{n}, m}\right\}_{m=0}^{\infty}$ is a multinorm on $\mathfrak{B}_{B, K_{n}, m}^{M}(I)$, a subspace of $\mathfrak{B}_{B}^{M}(I)$ consists of all hyperfunctions with support conained in $K_{n}$. The defined set of multinorms generates a topology $\tau_{K_{n}, m}$ on $\mathfrak{B}_{B, K_{n}, m}^{M}(I) . \mathfrak{B}_{B}^{M}(I)$ assigns the inductive limit topology $\tau$ when $K_{n}$ varies over all compact sets $K_{1}, K_{2}, \ldots$

Proposition 3.4. $\mathfrak{B}_{B}^{M}(I)$ is complete with respect to the inductive limit topology defined above

Proposition 3.5. $\mathfrak{B}_{B}^{M}(I)$ is a complete ordered linear topological space

Definition 3.6. The Positive Cone P of $\mathfrak{B}_{B}^{M}(I)$ when restricted to real valued hyperfunctions is the set of all non-negative hyperfunctions in $\mathfrak{B}(I)$. The positive cone in $\mathfrak{B}_{B}^{M}(I)$ is $P+i P$ which is denoted as $\mathscr{P}$

Proposition 3.7. The cone $\mathscr{P}$ in $\mathfrak{B}_{B}^{M}(I)$ has the following properties:

i) $\mathscr{P}+\mathscr{P} \subset \mathscr{P}$

ii)c $\mathscr{P} \subset \mathscr{P}$ for every real number $c$

iii) $\mathscr{P} \cap-\mathscr{P}=\{[0]\}$.
Proposition 3.8. $\mathscr{P}$ is a convex set in $\mathfrak{B}_{B}^{M}(I)$

Definition 3.9. For $f(x), g(x) \in \mathfrak{B}_{B}^{M}(I)$ with $f \leq g$, define the order interval between $f$ and $g$ by

$$
[f, g]=\left\{h(x) \in \mathfrak{B}_{B}^{M}(I): f(x) \leq h(x) \leq g(x)\right\}
$$

Definition 3.10. A subset $E$ of $\mathfrak{B}_{B}^{M}(I)$ is order bounded if there exists $f(x), g(x) \in \mathfrak{B}_{B}^{M}(I)$ such that $E \subset[f, g]$

Proposition 3.11. The cone $\mathscr{P}$ in $\mathfrak{B}_{B}^{M}(I)$ is generating.

Proof. Since every complex measurable function

$F=F^{+}-F^{-}$, we have $\mathscr{P}-\mathscr{P}=\mathfrak{B}_{B}^{M}(I)$

Definition 3.12. For $E \subset \mathfrak{B}_{B}^{M}(I)$ the full hull $[E]$ of $E$ is defined as

$$
[E]=\left\{h(x) \in \mathfrak{B}_{B}^{M}(I): f(x) \leq h(x) \leq g(x), f(x), g(x) \in E\right\}
$$

Proposition 3.13. The cone $\mathscr{P}$ in $\mathfrak{B}_{B}^{M}(I)$ is normal

Proof. The neighborhood basis of 0 for $\tau$ consisting of real analytic functions is a neighborhood basis of 0 consisting of full sets. Hence the cone $\mathscr{P}$ is a normal cone.

Proposition 3.14. Every order bounded subset of $\mathfrak{B}_{B}^{M}(I)$ is $\tau$ bounded

Proof. Proposition 1.4 in [2] states that if the cone $K$ in an ordered topological vector space $E(\tau)$ is normal for $\tau$, then every order bounded subset of $E$ is $\tau$-bounded.

Proposition 3.15. If $\mathscr{P}$ is a normal cone in $\mathfrak{B}_{B}^{M}(I)$ then $\mathscr{P} \cap \mathfrak{B}_{B, K_{n}, m}^{M}(I)$ is a normal cone in $\mathfrak{B}_{B, K_{n}, m}^{M}(I)$

Proof. By Proposition 1.8 of [2] if $K$ is a normal cone in an ordered topological vector space $E(\tau)$ and $M$ is a linear subspace of $E$ then $K \cap M$ is anormal cone in $M$ for the subspace topology.Result follows from this.

\section{Conclusion}

Using the order relation defined above in the space of hyperfunctions we can compare the hyperfunction solutions of partial differential equations. Also we can compare the inductive limit topology and order topology in the linear space of hyperfunctions.

\section{References}

[1] Urs Graf, Introduction to Hyperfunctions and Their Integral Transforms, Birkhauser, 2010.

[2] Peressini A L, Ordered Topological Vector Spaces, Harper and Row, New York, 1967.

[3] Geetha K V, Mangalambal N R, Stieltjes Transformation on Ordered Vector Space of Generalized Functions and Abelian and Tauberian Theorems,Int.J.Contemp.Math. Sciences, 3(33)(2008), 1619-1628. 
[4] Geetha K V, Mangalambal N R, The Laplace- Stieltjes Transformation on Ordered Topological Vector Space of Generalized Functions, Acta Math.Univ. Comenianae, Vol.LXXX(2)(2011), 243-249.

[5] A.H. Zemanian, Generalized Integral Transformations, Dover Publications, Inc., New York.

[6] Stevan Pilipovic, Bogoljub Stsnkovic, Convergence in the space of Fourier Hyperfunctions,Proc.Japan Acad.,73,Ser.A(1997), 33-35.

[7] B.Limaye, Functional Analysis, 3rd Edn., New Age International(P)Ltd., India.

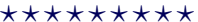 \\ ISSN(P):2319-3786 \\ Malaya Journal of Matematik \\ $\operatorname{ISSN}(\mathrm{O}): 2321-5666$

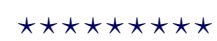

